\title{
El tratamiento institucional de la alimentación: un análisis sobre la intervención contra la obesidad
}

\author{
Cecilia Díaz-Méndez \\ Universidad de Oviedo. Departamento de Sociología \\ cecilia@uniovi.es
}

Recibido: 26-03-2010

Aceptado: 29-09-2011

\section{Resumen}

España tiene una de las tasas de obesidad infantil más altas de Europa. En este trabajo, se estudian las diferentes formas de afrontar institucionalmente el aumento de la obesidad en el entorno europeo en general y en España en particular. En él, se analizan las intervenciones para promover una dieta saludable, tanto las socioeducativas, como las orientadas a los contextos de consumo. La epidemia de la obesidad ha provocado un replanteamiento del papel de la alimentación en las instituciones. En primer lugar, ha pasado a ser un asunto social y ha dejado a un lado su tradicional carácter biológico. En segundo lugar, la alimentación se ha comenzado a tratar con políticas sanitarias, con lo que se ha alejado del tradicional tratamiento agrario. En tercer lugar, el aumento de la obesidad ha propiciado la corresponsabilidad en los problemas alimentarios entre el Estado y las industrias agroalimentarias.

Palabras clave: sociología de la alimentación; políticas alimentarías; consumo alimentario.

\section{Abstract. Institutional responses to dietary habits: An analysis of measures to combat obesity}

Spain has one of the highest childhood obesity rates in Europe. In this paper we study the different ways institutions are dealing with increasing obesity rates in Europe and in Spain in particular. We discuss measures taken to promote a healthy diet in both educational and consumer contexts. The obesity epidemic has led institutions to rethink the role of diet and nutrition. Firstly, diet has become a social issue and left aside much of its biological foundations. Second, diet has begun to form part of health policy agendas, moving away from the traditional agricultural-oriented viewpoint. Thirdly, the rise in obesity has led to corresponsibility between the state and food processing industries regarding food problems.

Keywords: sociology of food; food policy; food consumption. 


\title{
Sumario
}

\author{
1. Introducción \\ 3. La intervención sobre las estructuras \\ de consumo: regulación sobre la industria \\ 2. Perspectivas teóricas críticas sobre \\ la obesidad: formas diversas \\ de entender y detener la obesidad \\ agroalimentaria y control de la publicidad \\ 4. Conclusiones \\ Referencias bibliográficas
}

\section{Introducción}

La obesidad está siendo motivo de debate institucional y académico en toda Europa y ya ha sido denominada por la Organización Mundial de la Salud (OMS) como la epidemia no infecciosa del siglo XXI (OMS, 2004; OMS, 2006; CE, 2007) ${ }^{1}$. Los estudios realizados en los países desarrollados apuntan a un aumento sostenido del sobrepeso y de la obesidad en Europa y América. Los datos otorgan a España una de las tasas de obesidad infantil más altas de Europa. Los estudios comparados realizados entre 1975 y 2000 la colocan en primer lugar, puesto que se ha pasado de un $12 \%$ de sobrepeso y obesidad en 1980 a un 19\% en 1995 (Lobstein y Frelut, 2003). Estudios longitudinales posteriores, realizados entre países con datos del Panel Europeo de Hogares EUROSTAT, confirman un aumento sostenido del número de obesos en Europa entre 1998 y 2001, y los datos de la International Association for the Studie of Obesity (2007) muestran que España tienen unos porcentajes de obesos entre los 7 y los 17 años que le sitúan entre los primeros puestos. Según estos datos, en España son obesos el 13,9\% de los niños y el 8\% de las niñas entre 7 y 11 años; lo son también el 10\% de los niños y el 3\% de las niñas entre los 14 y los 17 años. Los datos del informe titulado Obesidad y economía de la prevención (OECD, 2010) muestran que la tasa de obesidad en España supera la media de los países de la OCDE. Así mismo, en las encuestas de salud del Ministerio de Sanidad, se confirma que, desde 1987 de la primera encuesta hasta la actualidad, la obesidad y el sobrepeso han ido en aumento. Según esta fuente, en el grupo de edad entre los 2 y los 17 años, tiene sobrepeso el 18,7\% y obesidad, el 8,9\%. Entre los grupos de edad de 18 y más años, la obesidad alcanza al 15,6\% de la población (INE, 2006).

Tanto en España como en el resto de Europa, los datos indican que la obesidad sigue creciendo, o que al menos no se frena como sería deseable, y es especialmente preocupante su aumento y su persistencia entre los grupos de menor edad (Costa-Font y Gil, 2008). Esto ha llevado a los analistas de diversos campos a reflexionar acerca de la intervención institucional intentando determinar el motivo del escaso éxito de las medidas adoptadas. La falta de evaluación de muchas de ellas está detrás de las reflexiones, pero los analistas

1. Así se ha constatado en los documentos de la Organización Mundial de la Salud: Estrategia global sobre dieta, actividad fisica y salud (2004) y Carta europea contra la obesidad (2006), así como en el Libro blanco sobre la obesidad, de la Unión Europea (2007). 
se han detenido a explicar teóricamente las formas de intervención y las consecuencias de las distintas estrategias adoptadas para frenar la obesidad. Los estudios existentes indican que el escaso éxito está directamente relacionado con el carácter parcial de las intervenciones. Por este motivo, se sostiene que solamente una visión global del problema y de la solución puede contribuir a reducir las actuales tasas de obesos (Lang, 2006). Para que esto sea posible es necesaria una intervención en la que se impliquen todos los agentes: Estado, empresas y ciudadanos.

En este trabajo, vamos a describir y comentar críticamente las diferentes formas de afrontar el aumento de la obesidad por parte de los poderes públicos en el entorno europeo en general y en España en particular. Vamos a comenzar presentando las diferentes posturas teóricas sobre las políticas contra la obesidad. A continuación, desarrollaremos los tipos de intervenciones en lo que podríamos denominar, de modo general, políticas alimentarias. Realizaremos un repaso de los objetivos de estas políticas y mostraremos algunos ejemplos españoles de las mismas, así como los resultados de la evaluación de este tipo de intervenciones. Esta revisión nos permite concluir que nos encontramos ante una nueva forma de comprender la alimentación desde el ámbito público. El origen de esta nueva visión se sitúa en el aumento de la obesidad, que ha tenido que ser afrontado por los poderes públicos como un problema social que ya no es posible ignorar por más tiempo.

\section{Perspectivas teóricas críticas sobre la obesidad: formas diversas de entender y detener la obesidad}

\subsection{Del análisis individual al tratamiento de la obesidad como asunto de salud pública}

Algunos investigadores consideraron en el pasado que los problemas de salud ligados a la alimentación, incluida la obesidad, tenían su origen en factores de carácter biológico (Neel, 1962). La intervención sobre la obesidad desde el punto de vista estrictamente biológico (al igual que otro tipo de medidas para afrontar enfermedades concretas o epidemias) requiere una intervención médica sobre las personas afectadas por la dolencia, las cuales deben reducir su peso. La expansión de la obesidad en las sociedades modernas ha puesto en cuestión estas orientaciones por dos motivos: en primer lugar, por considerar que la intervención sanitaria sobre la obesidad no muestra los resultados esperables sobre el individuo, pues no siempre se logra una reducción de peso sostenida (Cuevas y Reyes, 2005; Vázquez Martínez et al., 2008). En segundo lugar, y sobre todo, por considerar que la intervención, al ser de carácter individual, no tiene ningún impacto sobre la epidemia (Jain, 2005).

Estas perspectivas biológicas, aunque siguen marcando la intervención médica individual, son hoy excepcionales en el análisis de la obesidad y los propios estudios nutricionales han apostado desde hace décadas por la multicausalidad y por el carácter externo y social de las causas de la obesidad (Goldblatt 
et al., 1965). Crecen los adeptos hacia una consideración social del origen de la obesidad, especialmente en los entornos donde los problemas no están en las carencias alimentarias, sino en la sobreabundancia de alimentos. Así, la mayoría de los estudios nutricionales actuales ha incorporado un conjunto de variables de carácter socioeconómico al análisis de nutrientes. En España, la acción metodológicamente más relevante ha sido la vinculación institucional entre las encuestas de presupuestos familiares y las encuestas nutricionales realizada por el Instituto Nacional de Estadística. En varias oleadas, se ha propiciado la integración de variables de uno y otro campo, ofreciendo la posibilidad de relacionar el estado nutricional de la población española con las rentas familiares, con la situación ocupaciónal o con la estructura de los hogares (Varela y col., ENNA, 1971, 1985, 1995). Otros trabajos han completado esta orientación social incorporando a los estudios tradicionales el análisis de las creencias alimentarias (Vioque y Quiles, 2003). Además, de manera generalizada, ya se incluyen en las encuestas nutricionales preguntas sobre la práctica deportiva, la actividad física general en el tiempo libre, el origen social de los entrevistados o, como en la Encuesta Nacional de Salud, las percepciones de la población sobre su propia salud.

El primer avance para comprender el crecimiento de la obesidad y el sobrepeso en las sociedades modernas ha sido, por tanto, la incorporación de los aspectos sociales de la alimentación a los estudios «tradicionales» de carácter nutricional. Los analistas de los cambios nutricionales de la población coinciden al afirmar que es necesario afrontar el asunto desde una perspectiva de politica nutricional ${ }^{2}$ que promocione unos hábitos alimentarios acordes con las recomendaciones nutricionales y que permita tratar la obesidad como un asunto público (Serra Majen et al., 2007). Esta visión es compartida hoy por un gran número de científicos del ámbito sanitario y nutricional, así como de responsables de gestión política en las instituciones encargadas de la alimentación ${ }^{3}$.

Esta nueva forma de análisis ha presentado la obesidad como un problema social, con lo cual se traslada la responsabilidad a los poderes públicos, y deja en un segundo plano la visión estrictamente individual y médica del problema. Los datos son contundentes: la obesidad está ligada al origen social y son los grupos más desfavorecidos los que se ven más afectados por ella, tanto en España como en otras sociedades (Lhuissier and Regnier, 2005; Poulain, 2009; OCDE, 2010). Además, la obesidad genera un gran número de enfermedades y reduce la esperanza de vida de la población ${ }^{4}$. Y, derivado de este hecho, existe

2. Serra Majen, experto en obesidad infantil y responsable de gran número de estudios de este tipo en España, afirma: «[...] es necesaria una política de nutrición que sea efectiva en la promoción de una alimentación acorde con las recomendaciones nutricionales y la dieta mediterránea» (Serra Majen, 2007: 567).

3. Se puede leer la editorial de Félix Lobo: «Políticas públicas para la promoción de la alimentación saludable y la promoción de la obesidad», Rev.Esp..Salud Pública (2007), 5.

4. Las enfermedades a las que se suele asociar la obesidad son: la diabetes, la hipertensión arterial, el aumento de colesterol y de triglicéridos, el infarto de miocardio, la trombosis cerebral, la apnea del sueño, las enfermedades articulares y algunos tipos de cáncer. 
una clara relación entre el aumento de la obesidad y el crecimiento del gasto sanitario, aspecto confirmado tanto en España como en otros países (Finkelstein et al., 2005; Rodríguez Caro y González López-Valcárcel, 2009).

\subsection{La intervención socieducativa: programas formativos y difusión institucional de la información}

El paso de una visión individual y médica del problema a una más social y pública ha orientado la intervención contra la obesidad hacia el ámbito educativo, lo que ha dado a la solución un carácter preventivo. Este tipo de propuestas se sustenta en la constatación de los malos hábitos alimentarios entre la población infantil, generadores de sobrepeso y obesidad, tales como el consumo excesivo de azúcares, la falta de actividad deportiva o los malos desayunos, confirmados en estudios de ámbito nacional (Aranceta et al., 2003) y en las propias encuestas nacionales de salud ${ }^{5}$. Se justifica la intervención entre la población más joven por la fuerte relación entre la obesidad infantil y la obesidad en la edad adulta y se insiste en las enfermedades que puede provocar el sobrepeso y la obesidad en el futuro. Se opta así por una intervención con programas formativos e informativos dirigidos en particular a los menores y con una intervención preferente en los centros escolares. También se orienta la intervención a la población general a través de campañas divulgativas. Todo ello con el objetivo de modificar las actitudes de la población hacia la alimentación e inculcarle que siga una dieta saludable.

El programa PERSEO, del Ministerio de Sanidad, es un ejemplo de este tipo de intervenciones en centros escolares. Está dirigido al alumnado de entre 6 y 10 años. Su objetivo es "promover la adquisición de hábitos alimentarios saludables y estimular la práctica de actividad física regular entre los escolares, para prevenir la aparición de obesidad y otras enfermedades» ${ }^{6}$. Se entiende que para frenar la obesidad es preciso reeducar en hábitos alimentarios y estilos de vida que pueden estar dando lugar a pautas de comportamiento no saludables en una población, la infantil, que no debería, por sus condiciones de vida en las sociedades desarrolladas, tener este tipo de problemas. Una educación en buenos hábitos da lugar a una mejora de la capacidad de elección individual y se adiestra en hábitos saludables para el presente y para el futuro.

De modo general, en cualquier intervención de carácter educativo, existen importantes dificultades para confirmar su éxito, en parte porque sus resultados no siempre son medibles, pero también porque se precisan periodos de tiempo largos para corroborar su efectividad. Quizás por estas dificultades se cuenta con poca evaluación de los programas realizados y con frecuencia se

5. ENKID es un estudio nacional sobre el estado nutricional de la población infantil y juvenil de 2 a 24 años. En las encuestas de salud, aparecen datos sobre la práctica deportiva entre los jóvenes, el consumo de productos dulces o los alimentos ingeridos en los desayunos. Todos los datos apuntan a la necesidad de cambiar hábitos.

6. Se puede ver en http://www.perseo.aesan.msps.es/. 
desconocen sus efectos sobre la población intervenida ${ }^{7}$. Por supuesto, este tipo de intervenciones para frenar la obesidad son consideradas necesarias por todos los analistas, sin embargo, los pocos estudios realizados sobre su efectividad en el ámbito escolar muestran su limitado éxito (Ciriza et al., 2008). Otros trabajos han puesto de manifiesto que son más efectivas en poblaciones con niveles educativos altos y con ingresos altos (Swinburn y Egger, 2002). Estos son, precisamente, los grupos menos necesitados de orientación, si nos atenemos a la relación que existe entre la clase social y la obesidad. Como se sabe, son los grupos de estrato socioeconómico y educativo más bajo los más afectados por la epidemia (Lhuissier y Regnier, 2005; Aranzeta, 2008).

Además de utilizar programas de promoción de hábitos alimentarios, las administraciones públicas han recurrido a las guías alimentarias para la difusión de las dietas saludables, promovidas por los organismos internacionales. A través de ellas, se difunden entre la población los estándares de salud nutricional vigentes. Todos los países han desarrollado guías especiales y han ofrecido, a lo largo de la historia reciente, orientaciones de este tipo (Gifford, 2002). En España, también han tenido su propio desarrollo desde la creación del código alimentario español y la difusión de normas a través de la rueda de los alimentos, en los años setenta (Díaz-Méndez y Gómez-Benito, 2010).

Pero las críticas a estas formas de promoción han sido más contundentes que las realizadas a las intervenciones educativas, pues, al analizar los cambios en las recomendaciones con perspectiva histórica, se ponen en evidencia sus contradicciones y también sus escasos éxitos. Así, por ejemplo, los programas de racionamiento de la Segunda Guerra Mundial dieron buenos resultados y las recomendaciones fueron seguidas por una población necesitada de alimentos, pero esto no fue tan exitoso en años sucesivos y, por ejemplo, las recomendaciones para disminuir el consumo de grasa en Estados Unidos en los años noventa coinciden con un aumento del consumo de grasa entre la población (Gifford, 2002). Sucede algo similar en el estudio de las recomendaciones de las administraciones españolas. Las realizadas en los años sesenta, orientadas a introducir variedad en una dieta tradicional muy monótona tuvieron más éxito que las que sugirieron en los noventa retomar los consumos de legumbres de los platos tradicionales (Díaz-Méndez y Gómez-Benito, 2010). A modo de ejemplo, en los años setenta, el Instituto Nacional de Consumo español decía: «Multitud de autores han demostrado que el pescado congelado tiene un valor nutritivo superior al que habitualmente llamamos fresco" y, en 1985, se decía, en las charlas del programa EDALNU de Educación Nutricional: «El pan blando se digiere mejor que el integral, pues su contenido en fibra es más bajo y, en consecuencia, mejor tolerado» (referenciado en Díaz-Méndez y Gómez-Benito, 2008). Ambas recomendaciones serían hoy consideradas poco correctas o cla-

7. La Agencia Española de Seguridad Alimentaria y Nutrición organiza periódicamente una convención sobre los resultados de la Estrategia NAOS. En ellas, se analizan los resultados de intervenciones concretas en la lucha contra la obesidad en las diferentes comunidades autónomas españolas. 
ramente inapropiadas, pero adquieren sentido al relacionarlas con la necesidad de promocionar el consumo de estos productos entre la población española del momento histórico en el que se emiten. Por eso algunos analistas han afirmado que las recomendaciones alimentarías son construcciones sociales ligadas a un determinado momento histórico y a grupos de expertos con intereses concretos, no siempre coincidentes con el bienestar general de la población (Smith et al., 2000; Nestle, 2002; Díaz-Méndez y Gómez-Benito, 2010).

Para explicar la falta de efectividad de las guías alimentarias, algunos autores afirman que los consumidores tienen problemas para comprenderlas y, por lo tanto, para seguir correctamente sus recomendaciones. Si las guías se basan en nutrientes pero el consumidor come alimentos, es lógico que exista una cierta distancia entre lo que se recomienda y lo que se consume (Asp, 1999; Gifford, 2002; Grunert et al., 2010). Otros estudios ponen el acento en la multiplicidad y la contradicción de los mensajes que se emiten como factor explicativo de su escasa efectividad. Al proceder de departamentos diferentes, con intereses diferentes (los departamentos de agricultura y los de salud y consumo son los más habituales), la información que llega al consumidor no es la apropiada. Los mensajes son confusos y esto impide tomar decisiones concordantes con los estándares nutricionales (Gifford, 2002; Nestle, 2002).

Es cierto, además, que el propio conocimiento científico sobre los alimentos y sus propiedades se manifiesta cambiante y que las recomendaciones varían en función de los avances de las ciencias de la salud y la alimentación. Menéndez Patterson (2008) sostiene que sería necesario actualizar conceptos e ideas sobre nutrición que se arrastran desde principios del siglo pasado y que, a veces, dificultan y encorsetan el desarrollo de esta ciencia. La propia definición de obesidad no es fácilmente delimitable, y hay ciertas reticencias entre los expertos a su uso entre la población infantil y juvenil (Aranceta, 2008). Estos son solamente algunos ejemplos de la complejidad que entraña establecer una frontera entre lo que se considera incorrecto en términos científicos y lo que no lo es, aún siendo el estado el que adopte el rol de garante de lo apropiado.

\section{La intervención sobre las estructuras de consumo: regulación sobre la industria agroalimentaria y control de la publicidad}

La perspectiva adoptada desde la intervención formativa e informativa se sostiene en la consideración de que la responsabilidad sobre los problemas alimentarios está, principalmente, en las personas afectadas, dado que ellas (o sus familias) deciden sobre la forma en que se nutren. Los individuos son los responsables de su alimentación, de su peso y, por tanto, de dejar de ser obesos. Pero se ha planteado también la necesidad de considerar que las decisiones se toman en entornos alejados del ámbito escolar y familiar y fuera del control de la intervención individual. En estos casos, se responsabiliza a los denominados entornos obesogénicos. Desde esta perspectiva, se esgrime que las pautas alimentarias inapropiadas que conducen a hábitos de vida poco saludables y producen obesidad y sobrepeso son conductas impuestas desde fuera del individuo y 
ajenas a la voluntad de los afectados. Comer de manera sana supone enfrentarse diariamente a espacios mercantilizados en los cuales se toman las decisiones de consumo y, por lo tanto, es en estos entornos de consumo en los que se propicia una alimentación que favorece la obesidad (Swinburn y Egger, 2002). En definitiva, se entiende que el análisis de la obesidad es indisociable de un entorno de lógicas industriales y económicas decisivas para la producción y el mantenimiento de los malos hábitos alimentarios (Luque, 2008; Jain, 2005; Lang y Rayner, 2007) y se responsabiliza a las empresas agroalimentarias. Este entorno obesogénico estimula al consumidor para que seleccione los productos alimentarios en función de criterios alejados de la racionalidad nutricional aconsejable y le impide seguir hábitos de vida saludables. En unos casos, porque los productos no son realmente saludables, en otros, porque se estimula el consumo no saludable a través de la publicidad.

\subsection{Intervención sobre la industria: regulación de la agroindustria $y$ de la publicidad}

Una de las vías de intervención pública sobre los factores estructurales del consumo alimentario ha sido la de regular la acción de las empresas agroalimentarias. El planteamiento del que se parte para ello es generar un entorno de consumo saludable desde el punto de vista de la oferta, de modo que la presencia en el mercado de productos sanos incentive su consumo entre la población (Bush y Williams, 1999, Smith et al., 2000; Chandon y Etile, 2010). Este tipo de intervenciones tiene diferentes orientaciones que se podrían clasificar, de modo general, en dos tipos: la que actúa sobre la propia actividad empresarial, bien regulando sus procesos de trabajo o la publicidad, y la que incide sobre el sector de la producción de alimentos.

Aunque históricamente la alimentación ha sido objeto de cierta regulación, es desde la segunda mitad del siglo XX cuando ha alcanzado un desarrollo y una expansión significativa. La intervención sobre la industria en el inicio y el desarrollo de la sociedad industrial moderna ha tenido por objetivo la regulación de la oferta para garantizar una alimentación segura. Al inicio del siglo XXI, la intervención ha tomado un cariz distinto, con una orientación más volcada en la salud, aunque la frontera entre lo seguro y lo sano sea difusa (León Arce, 2008). Los trabajos que analizan los cambios en la legislación alimentaria española muestran que las políticas de seguridad alimentaria han sido una respuesta de regulación para proteger al consumidor, aunque esto se haya producido en ocasiones después de que los problemas se hayan materializado (León Arce, 2008). Sea como fuere, la industria agroalimentaria se convierte en foco de regulación institucional frente a un consumidor vulnerable y necesitado de protección ante las recurrentes crisis agroalimentarias.

Pero la acción institucional sobre el entorno de consumo más conocida es la orientada a regular la publicidad. La publicidad alimentaria representa la fuente principal de información sobre los alimentos que ofrece la industria alimentaria. Entre los trabajos españoles que analizan la relación entre la publicidad y la 
alimentación (Gracia, 1996; Díaz-Méndez y González, 2008), se constata que las empresas agroalimentarias han ofrecido y ofrecen sus productos con estrategias de venta que logran acoplar sus intereses con los deseos del consumidor, al igual que se ha hecho con otro tipo de productos en el marketing tradicional. No es difícil rastrear cómo en algunas épocas las golosinas se han ofrecido como alimento, la tradición como valor asociado a productos industriales transformados y la delgadez como referente estético de salud (Díaz-Méndez y González, 2008). Y los estudios confirman la relación entre la publicidad de alimentos y sus efectos perniciosos sobre el aumento de peso entre la población infantil y en sus hábitos alimentarios (Halford et al., 2008; Harris et al., 2009; Menéndez García y Franco, 2009). La forma de ofrecer los alimentos en la publicidad, fuertemente anclada en los deseos de los consumidores, propicia que la oferta alimentaria se desligue de la racionalidad que caracterizaría una dieta considerada adecuada por los nutricionistas. Entre el consumidor y los productos alimentarios publicitados, se crea un entorno en el que el gusto y la salud no siempre son coincidentes (Contreras y Gracia, 2008). Contreras constata que lo que denomina pirámide del gusto, en referencia a la clasificación de los alimentos en función del placer que proporcionan al paladar, no coincide con la pirámide nutricional procedente de las recomendaciones nutricionales, ni tampoco coincide lo que se come realmente con lo que se recomienda como saludable.

La Administración española ha promovido la adscripción de las empresas al denominado Código $\mathrm{PAOS}^{8}$, que supondría una referencia ética a la hora de diseñar la publicidad de alimentos con el objetivo de afrontar el aumento de la obesidad infantil. La mayoría de las empresas agroalimentarias españolas se ha adherido a esta iniciativa, aunque las evaluaciones sobre el cumplimiento del Código PAOS indican que no ha tenido mucha repercusión, ni sobre la cantidad ni sobre la calidad de los anuncios de alimentos destinados al público infantil (Menéndez y Franco, 2009).

\subsection{Intervenir sobre la producción}

Otra de las vías de la intervención para afrontar el aumento de la obesidad ha sido la intervención sobre la producción. En general, las orientaciones sobre la producción tratan de promover el aumento del consumo de ciertos productos apoyando económicamente su producción mediante subvenciones gubernamentales. Se fundamentan en el supuesto de que el abaratamiento de costes genera un aumento de productos saludables y baratos en el mercado que ayudan a los consumidores a encontrarse con más y mejores opciones de consumo saludable. Es una orientación que en algunos países ha estado dirigida expresamente hacia los grupos más desfavorecidos y más afectados por la obesidad y que suelen optar por productos más baratos en sus compras. Esta orientación

8. Se ha desarrollado dentro de la estrategia NAOS y en colaboración con la Organización Mundial de la Salud y las instituciones comunitarias a partir del año 2005. 
se ha desarrollado en particular en Estados Unidos, donde la epidemia de la obesidad ha llegado antes que a Europa (Bush y Williams, 1999). También se ha intervenido reduciendo los impuestos de los productos considerados más saludables, por ejemplo: los empty calories, o imponiendo tasas a los menos saludables, en general por exceso de azúcar o grasas (Nestlé y Jacobson, 2000; Epstein et al., 2010; Allais, Bertail y Nichele, 2010). Esta orientación también se propone en la Ley de seguridad alimentaria y nutrición española aprobada en marzo de 2011.

Todos parecen estar de acuerdo en que este tipo de relaciones entre los incentivos a la producción y la modificación en las pautas de consumo debería potenciarse aún más (Duxbury y Welch, 1999). Sin embargo, las evaluaciones de los programas no son totalmente positivas. Así, por ejemplo, el consumo de carne en Estados Unidos aumenta al bajar su precio por las ayudas financieras del gobierno a la producción, pero también con ello aumenta el consumo de grasas que alejan a la población de los estándares nutricionales de salud (Kinsey y Bowland, 1999). Los resultados del aumento de impuestos a los productos grasos en Francia indica algo similar, un efecto muy pequeño a corto plazo sobre las compras de los hogares, y aunque el efecto sobre el peso es algo mayor a largo plazo, los autores no parecen optimistas respecto a sus resultados para reducir la obesidad (Allais et al., 2010).

Fuera de las fronteras de la Unión Europea, la mayoría de estas políticas agroalimentarias han tenido un importante desarrollo con el objetivo de afrontar el aumento de la obesidad, pero no ha sido así en España. Realizando un recorrido histórico de las políticas agroalimentarias españolas desde los años sesenta hasta la actualidad, se puede afirmar que, a lo largo del siglo XX, la relación entre el Estado y las empresas agroalimentarias ha estado marcada por una fuerte colaboración institucional que ha favorecido la expansión y el desarrollo de la industria agroalimentaria española (Gómez-Benito, 2004). Se puede constatar un apoyo institucional fuerte a la producción agroalimentaria en los inicios del desarrollo agrario español, durante la llamada revolución verde y con el soporte de los programas de desarrollo agrícola y ganadero del franquismo. Son bien conocidos los planes de regadío o la colonización agraria de la época franquista. La entrada de España en la Comunidad Europea supuso una promoción específica de algunos productos nacionales emblemáticos en el mercado español que buscaron, con apoyo institucional, un lugar en los mercados internacionales. Así sucedió con el aceite, que se vio respaldado por las campañas institucionales como "Conozca los alimentos», del Instituto Nacional de Consumo en 1978, o, posteriormente y hasta la actualidad, con la campaña «Productos de España», desarrollada por el Ministerio de Agricultura desde 1997. La promoción de productos locales, como los plátanos de Canarias o los mejillones gallegos, contaron y cuentan con el soporte gubernamental. Y, más recientemente, la colaboración de las empresas y el Estado con las asociaciones de viticultores, de pescadores o de otros grupos de pequeñas o grandes empresas muestra la relevante colaboración institucional del Estado a lo largo de diferentes períodos históricos (Díaz-Méndez y Gómez-Benito, 2008). En 
el año 2005, se ha puesto en marcha la estrategia NAOS, un plan de acción contra la obesidad infantil que promueve la Agencia Española de Seguridad Alimentaria y Nutrición y al que se han adherido la mayoría de las industrias agroalimentarias españolas (Ballesteros, 2007). Se puede afirmar que los intereses de los productores agrarios han contado con el respaldo del Estado a lo largo del desarrollo de la moderna agroindustria, aunque la promoción haya tenido más un carácter económico empresarial de apoyo a la industria y solo recientemente se ha planteado como una intervención para generar entornos de consumo saludables que implique también a las industrias en los problemas derivados de una mala alimentación.

\section{Conclusiones}

La epidemia de la obesidad ha sido la principal responsable del replanteamiento del papel de la alimentación en las instituciones públicas. El cambio en el rol de la alimentación se vislumbra en tres direcciones.

En primer lugar, las instituciones han comenzado a abandonar la inicial orientación biológica e individual y a considerar la más reciente visión social del problema de la obesidad. Aunque no se ha abandonado el tratamiento médico e individual del problema, los poderes públicos trabajan ya con una concepción multicausal de la obesidad y actúan preferentemente sobre los aspectos que tiene que ver con el origen social de las causas que la provocan. Los problemas alimentarios, pero muy especialmente la obesidad, se tratan como un asunto de carácter público.

En segundo lugar, se está dando un nuevo tratamiento institucional a la alimentación que hace que se vincule más al ámbito sanitario y se aleje del ámbito agrario. La separación histórica entre las políticas agrarias y las políticas nutricionales se ha roto. Aunque aún las políticas siguen estando parceladas y es manifiesta la distancia entre las propiamente agrarias y las exclusivamente sanitarias, la necesidad de afrontar el aumento de la obesidad ha propiciado una confluencia de intereses y una aproximación de las intervenciones favorecida por la creación de instituciones, como la Agencia Española de Seguridad Alimentaria y Nutricional, que buscan la confluencia de objetivos para hacer descender la obesidad infantil.

En tercer lugar, se ha ampliado la responsabilidad en la preservación de la salud de los ciudadanos hacia los agentes privados implicados en la cadena agroalimentaria, lo cual complementa el papel del Estado en la promoción de hábitos alimentarios saludables.

Este nuevo rol de la alimentación ha propiciado una orientación multisectorial de las intervenciones públicas para frenar la obesidad que no está exenta de problemas. Tanto la formación y la información, como la intervención sobre la publicidad, la producción o la industria, son vías consideradas necesarias y complementarias para frenar la obesidad por parte de todos los analistas. Intervenir considerando prioritaria la población más joven parece apropiado, por tratarse de los grupos más vulnerables. Sin embargo, la uniformidad de las 
intervenciones socioeducativas e informativas, que no diferencian entre grupos de mayor o menor riesgo y homogeneiza las propuestas, puede ser un problema para alcanzar buenos resultados. La escasez de trabajos que evalúen los efectos es también un importante inconveniente para conocer sus éxitos o sus fracasos.

Las intervenciones sobre el contexto se han planteado desde una perspectiva integral del problema y ese es su mayor potencial, pues tienen en cuenta los diferentes agentes de la cadena agroalimentaria. Así, la intervención sobre la industria o la publicidad, o la conexión de las administraciones con agentes privados, pone en evidencia el interés por implicar a estos agentes en la mejora de la situación alimentaria de la población y asumir su parte de responsabilidad en los problemas de salud. Sin embargo, podemos considerar dos aspectos cuestionables. Por un lado, la escasa intervención sobre la producción agroalimentaria, que si bien es un sector fuertemente regulado, esta regulación no está orientada a la mejora de los hábitos alimentarios y la reducción de la obesidad. Por otro lado, la creciente regulación de la industria agroalimentaria, que respalda a un consumidor necesitado de un entorno de consumo saludable, pero que puede constituir un freno al desarrollo económico de estos sectores en un contexto de globalización alimentaria en mercados menos restrictivos que el europeo.

La obesidad sigue aumentando, pero también la intervención institucional para ponerle freno y la reflexión académica sobre sus causas, sus consecuencias y las formas de reducirla. Se ha avanzado claramente en adoptar una visión social del problema, y se reflexiona ya sobre el escaso éxito de algunas de las intervenciones que apuntan a una mayor conexión entre los intereses del Estado, las empresas y los ciudadanos.

\section{Referencias bibliográficas}

Allais, Olivier; Bertail, Patrice y Nichele, Véronique (2010). «The Effects of a Fat Tax on French Households' Purchases: A Nutritional Approach». American Journal of Agricultural Economics, 92 (1), 228-245.

ARANCETA, Javier (2008). «Obesidad infantil: nuevos hábitos alimentarios y nuevos riesgos para la salud». Alimentación, Consumo y Salud, 24, 216-245.

ARANCETA, Javier et al. (2003). «Sociodemographic and lifestyle determinants of food patterns in Spanish children and adolecents: The ENKIDS study». European Journal of Clinical Nutrition, 57 (1), 540-544.

ARANCETA Javier; SERRA MAJEM, Lluis (eds.) (2001). Obesidad infantil y juvenil: Estudio enkid. Barcelona: Masson.

AsP, Elaine (1999). «Factors affecting food decisions made by individual consumers». Food Policy, 24 (2-3), 287-294.

BALlesteros ArRibAS, Juan Manuel et al. (2007). «La estrategia para la nutrición actividad física y prevención de la obesidad». Revista Española de Salud Pública, $81(5), 443-449$.

Bush, Laina y WiLliams, Richard (1999). Diet and health: New problems/new solutions. Food Policy, 24, 135-144.

CE (2007). Libro blanco sobre la obesidad. 
Chandon, Pierre y ETILe, Fabrice (2010). Comportements alimentaires et politiques de santé nutritionnelle: Prix, information, marketing: quelles régulations? Working Paper ALISS 2010-05, INRA.

Ciriza, Edurne; PÉrez Rodrigo, Carmen y ArAnCETA, Javier (2008). «La difícil tarea de promocionar el consumo de frutas y verduras en el medio escolar: una revisión sistemática». Revista Española de Nutrición Comunitaria, 14, 6-20.

Collins, Keith (1999). «Public policy and the supply of food». Food Policy, 24, 311 324.

Contreras, Javier y Gracia, Mabel (2008). «Preferencias y consumos alimentarios: entre el placer, la conveniencia y la salud». Alimentación Consumo y Salud, 24, 153-188.

Costa-FONT, Joan y GIL, Joan (2008). "What lies behind socio-economic inequalities in obesity in Spain: a decomposition approach». Food Policy, 33 (1), 61-73.

Cuevas, Ada y Reyes, María Soledad (2005). «Lo último en diagnóstico y tratamiento de la obesidad. ¿Hay lugar aún para la terapia conservadora?». Rev. Med. Chile, $1333,713-722$.

Díaz Méndez, Cecilia y Gómez Benito, Cristóbal (2008). «Evolución de las recomendaciones institucionales sobre salud y alimentación». Alimentación, Consumo y Salud, 24, 25-50.

- (2010). «Nutrition and the Mediterranean diet: A historical and sociological analysis of the concept of "health diet" in Spanish society". Food Policy Journal, 35, 437-447.

Díaz MÉndez, Cecilia y GonzÁlez, María (2008). «Industria y alimentación: De la publicidad referencial a los alimentos funcionales». Alimentación, Consumo y Salud, 24, 105-130.

Duxbury, John M. y WelCH, Ross M. (1999). «Agriculture and dietary guidelines». Journal of Food Policy, 24 (2-3), 197-209.

EPSTEIN, Leonard H. et al. (2010). "The influence of taxes and subsidies on energy purchased in an experimental purchasing study». Psychological Science, 5 (febrero).

Finkelstein, Eric A.; Ruhm, Christopher y KosA, Katherine M. (2005). «Economic causes and consequences of obesity». Annual Review of Public Health, 26, 239-257.

GIFFORD, David K. (2002). «Dietary fats, Ealing guides, and public policy: History, critique, and recommendations». The American Journal of Medicine, 13 (9B), 89-113.

GoldBlatT, Phillip B.; MoOre, Mary E. y STURKARD, Albert J. (1965). «Social factors in obesity». The Journal of American Medical Asociation, 192, 1039-1044.

Gómez BeniTo, Cristóbal (2004). «Una revisión y una reflexión sobre la política de colonización agraria en la España de Franco». Historia del Presente, 3, 65-86.

Gracia, Mabel (1996). Paradojas de la alimentación contemporánea. MAPA.

GRUNERT, Klaus G. et al. (2010). «Use and understanding of nutrition information on food labels in six European countries». Journal of Public Health, 18 (3), 261-277.

HALFORD, Jason C. et al. (2008). «Beyond-brand effect of television food advertisenents on food choice in children: The effect of weight status». Public Health Nutrition, 11 (9), 897-904.

HARrIS, Jennifer L.; BARGH, John A. y Brownell, Kelly D. (2009). «Priming Effects of Television Food Advertising on Eating Behavior». Health Psychol, 28 (4), 404-413.

INE (2006). Encuesta Nacional de Salud. Madrid.

JAIN, Anjali (2005). «Treating obesity in individuals and population». Clinical Review BMJ, 331, 1387-90 
LANG, Tim (2006). «Food, the law and public health: three models of the relationships». Public Health, Journal of the Royal Institute of Public Health, 120, 30-41.

LANG, Tim y RAYNER, Geoff (2007). «Overcoming policy cacophony on obesity: an ecological public health framework for policymakers». Obesity Reviews, 8 (1), 165-181.

KINSEY, Jean y BOWLAND, Brandford (1999). «How can the US food system deliver food products consistent with the dietary guidelines?: Food marketing and Retailing: an economic view». Food Policy, 24, 237-253.

LHUISSIER, Anne y REgnier, Faustine (2005). "Obesity and food in working classes: An approach of the femanle body». CORELA, INRA, 3-4 (diciembre).

LUQUE, Emilio (2008). «La obesidad más allá del consumidor: Raíces estructurales de los entornos alimentarios». Alimentación, Consumo y Salud, 24, 130-149.

LEÓN ArCE, Alicia (2008). «Políticas alimentarias y seguridad del consumidor». Alimentación, Consumo y Salud, 24, 81-104.

LOBO, F. (2007). «Políticas públicas para la promoción de la alimentación saludable y la promoción de la obesidad». Rev.Esp.Salud Pública, 5.

LobSTEIn, Tim y Frelut, Marie-Laure (2003). «Prevalence of overweigtht among children in Europe. International». Obesity Reviews, 4, 195-200.

Menéndez García, R.A. y Franco Díez, F.J. (2009). «Publicidad y alimentación: Influencia de los anuncios gráficos en las pautas alimentarias de infancia y adolescencia». Nutrición Hospitalaria, 24 (3), 318.

MenÉNDEZ PATterson, Ángeles (2008). «Avances científicos en alimentación y nutrición». Alimentación, Consumo y Salud, 24, 55-79.

NeEL, James V. (1962). "Diabetes mellitus: a "thrifty" genotype rendered detrimental by "progress" ". Am J. Hum Genet, 14, 353-362.

NestLe, Marion (2002). Food politics: How the Food Industry Influences Nutrition, and Health. California. University of California Press.

Nestle, M. y Jacobson, Michael (2000). "Halting the obesity epidemic: A public health policy approach». Public Health Reports, 115 (1), 12-24.

OCDE (2010). Obesidad y economía de la prevención.

OMS (2004). Estrategia global sobre dieta, actividad fisica y salud.

- (2006). Carta europea contra la obesidad.

Poulain, Jean Pierre (2009). Sociologie de l'obésité. París: PUF.

Rodríguez CARo, Alejandro y GONZÁlez López-VALCÁrCEL, Beatriz (2009). «El trasfondo económico de las intervenciones sanitarias en la prevención de la obesidad». Revista Española de Salud Pública [en línea], 83 (1).

Smith, Lisa C.; AmAni, El Obeid y Jensen, Hellen H. (2000). «The geography and causes of foof insecurity in developing countries». Agricultural Economics, 22, 199-215.

Serra Majen, Lluís y otros (2007). «Tendencias del estado nutricional de la población española: Resultados del sistema de monitorización nutricional de Cataluña (19922003)». Revista Española de Salud Pública [en línea], 81, 5.

SwINBURN, Boyd y EGGER, Garry (2002). «Preventive strategies against weight gain and obesity: The international association for the study of obesity». Obesity Reviews, 3, 289-301.

VARELA, Gregorio et al. (varios años). Encuestas nacionales de nutrición y alimentación (ENNA, 1971, 1985, 1995)5, INE.

VÁzQUez MARTíneZ, Clotilde et al. (2008). "Una sentencia firme contra la mala práctica en el tratamiento de la obesidad». Nutrición Hospitalaria, 23 (3), 177-182.

VIOQUE, Jesús y QUILES, Joan (2003). Encuesta de Nutrición y Salud de la Comunidad Valenciana, 1994. Alicante. 